\title{
An Amplitude-Based Modeling Method and its Application on the Impedance Inversion in Heterogeneous Paleokarst Carbonate Reservoirs
}

\author{
Yuanyin Zhang ${ }^{1,2,3,4}$, Zandong Sun ${ }^{4,5}$, Zhijun Jin ${ }^{1,2,3}$, Ning Dong ${ }^{1,2,3}$, Yequan Chen ${ }^{1,2,3} \&$ Xiwu Liu ${ }^{1,2,3}$ \\ ${ }^{1}$ Petroleum Exploration and Production Research Institute, SINOPEC, Beijing, China \\ ${ }^{2}$ Sinopec Key Laboratory of Shale Oil/Gas Exploration and Production Technology, Beijing, China \\ ${ }^{3}$ National energy R \& D center of shale oil, Beijing, China \\ ${ }^{4}$ China University of Petroleum-Beijing, Beijing, China \\ ${ }^{5}$ PetroChina Tarim Oilfield Co, Korla, China \\ Correspondence: Yuanyin Zhang, Petroleum Exploration and Production Research Institute, SINOPEC, Beijing, \\ China. 100083. Tel: 86-010-8231-2090. E-mail: yuanyinshou@163.com
}

Received: February, 2016

doi:10.5539/esr.v5n2p199
Accepted: July 22, 2016

Online Published: July 30, 2016

\begin{abstract}
For the modeling of complex reservoirs with strong heterogeneity, for instance the deeply buried paleokarst reservoirs in the Tarim Basin, the traditional method by lateral interpolation and extrapolation of measured logs between well locations with the guiding of interpreted seismic horizons is driven by distance and often leads to non-geologic solutions, while the past improvements via adding seismic velocities or attributes information are still not accurate due to the resolution limitation or AVO (amplitude versus offset) effects contamination. In this paper, we present an amplitude-based modeling method by utilizing the heterogeneous information from seismic data to guide the geological model construction, based on the inverted pure P-wave data which have removed the AVO effects. The proposed method is applied in the impedance inversion of the paleokarst carbonate reservoirs in the Tarim Basin, where the reservoirs are characterized by substantial heterogeneity. Both the constructed Low frequency model (LFM) and the inverted impedance results of proposed method are more correlative with drilling data than that of traditional method. This method is more beneficial for strong heterogeneous reservoirs description especially in well insufficient or absent areas, suggested by the comparisons with traditional methods in the ZG8 area.
\end{abstract}

Keywords: Carbonate; amplitude-based modeling; P-wave data; heterogeneity; Tarim Basin

\section{Introduction}

Seismic data are routinely inverted into the relative impedance to provide quantitative predictions of reservoir properties-such as porosity, lithology fluid content etc., under specific conditions (Cerney \& Bartel, 2007, Pedersen-Tatalovic et al., 2008). However, the seismically inverted relative impedance only has a limited bandwidth depending on the band-limited input seismic data, comparing with the measured well logging data or in a laboratory. It has to be further transformed into the true absolute impedance for reservoir properties predictions away from well control, via merging reliably low-frequency information of the constrained low frequency model (LFM) (Cerney \& Bartel, 2007; Pedersen-Tatalovic et al., 2008; Zou et al., 2013; Grant, 2013). In essence, the constrained LFM represents the initial prediction to construct the inversion and helps to result in a better resolvability or link between the seismic data and the actual geology. Thus, the final inverted result of predicted sediment properties is actually a set of elastic volumes that deviate as little as possible from the initial predictions, while at the same time modeling the real geology as closely as possible (Hampson et al., 2005; Huck et al., 2010). The traditional LFM building method by lateral interpolation and extrapolation of impedance logs between well locations with the guiding of interpreted seismic horizons through a variety of mathematical methods such as weighted inverse-distance, spline, and kirging etc., is driven by distance and often leads to non-geologic solutions (Cerney \& Bartel, 2007; Pedersen-Tatalovic et al., 2008; Zou et al., 2013; Grant 2013). Although seismic velocities can be combined to partially provide the missed low frequency information, it is 
limited by the low resolution and accuracy which generally deteriorate with the increase of the burial depth. Meanwhile, seismic attributes are gradually employed into the construction of LFMs to improve the inversion reliabilities (Schultz et al., 1994; Liu et al., 2010; Pedersen-Tatalovic et al., 2008; Zou et al., 2013), but the conventional full-stack data and corresponding attributes are reported to be contaminated by the AVO (amplitude versus offset) effects no matter how much signal to noise ratio (SNR) degree can be enhanced via stacking (Zhang et al., 2013).

In the Tarim Basin in Western China, Karstified Ordovician limestone has been shown to be a highly productive oil and gas reservoir (Sun et al., 2011; Yang et al., 2012). The Ordovician carbonate reservoirs in this field are deeply buried $(>5000 \mathrm{~m})$ (Yang et al., 2010) and characterized by strong heterogeneity and some degree of anisotropy. These reservoir spaces are formed by a product of complex karstification related to unclear paleokarst drainage systems and subsequent complicated tectonic disruptions during burial (Zeng et al., 2011; Sun et al., 2012; Zhang et al., 2011a; Zhang et al., 2011b). To reasonably describe this strong heterogeneous reservoir and hence achieve accurate properties for prediction, we propose an amplitude-based modeling method by utilizing the heterogeneous information from seismic data to guide the geological model construction, based on the inverted pure P-wave data which have removed the AVO effects. The proposed method is applied in the impedance inversion of the paleokarst carbonate reservoirs in the ZG8 area, Tarim Basin.

\section{Background geology}

With two strong tectonic movements during the Early and Late Paleozoic, the Ordovician carbonate rocks experienced strong weathering and erosion and formed a karst reservoir, which is overburdened by Carboniferous and Triassic clastic rocks. The present burial depth of the karst reservoirs in the Tarim Basin is greater than $5000 \mathrm{~m}$ (Yang et al., 2010). Because of this deep burial and compaction, large voids that are associated with paleokarst chambers would be unusual in the Ordovician sequence because these features generally collapse and disappear beyond $3000 \mathrm{~m}$ of burial (Loucks, 1999). In addition, core analyses indicate that the background porosity in conventional cores is extremely low $(<2 \%)$, and the storage spaces are dissolution pores and fractures which are dominated by visible dissolution caves, holes, and fractures (Figure. 1a) (Sun et al., 2012). Consequently, widespread strong reflection amplitudes that are related to high reflection coefficients at brine-hydrocarbon fluid contacts, which are typical in shallow porous unconsolidated reservoirs, are not expected (Zeng et al., 2011). On the contrary, anomalously strong seismic amplitudes that frequently occur in the Middle and Lower Ordovician succession beneath the top Ordovician unconformity are characteristic of the Tarim Basin. These anomalous amplitudes (i.e., the 'bead-like reflections' (BRs, often called lamb kebabs) in the post-stack section (Figure. 1c)) are associated with high-velocity limestone matrix and low-velocity reservoirs and are often regarded as the best indicator of good reservoirs.
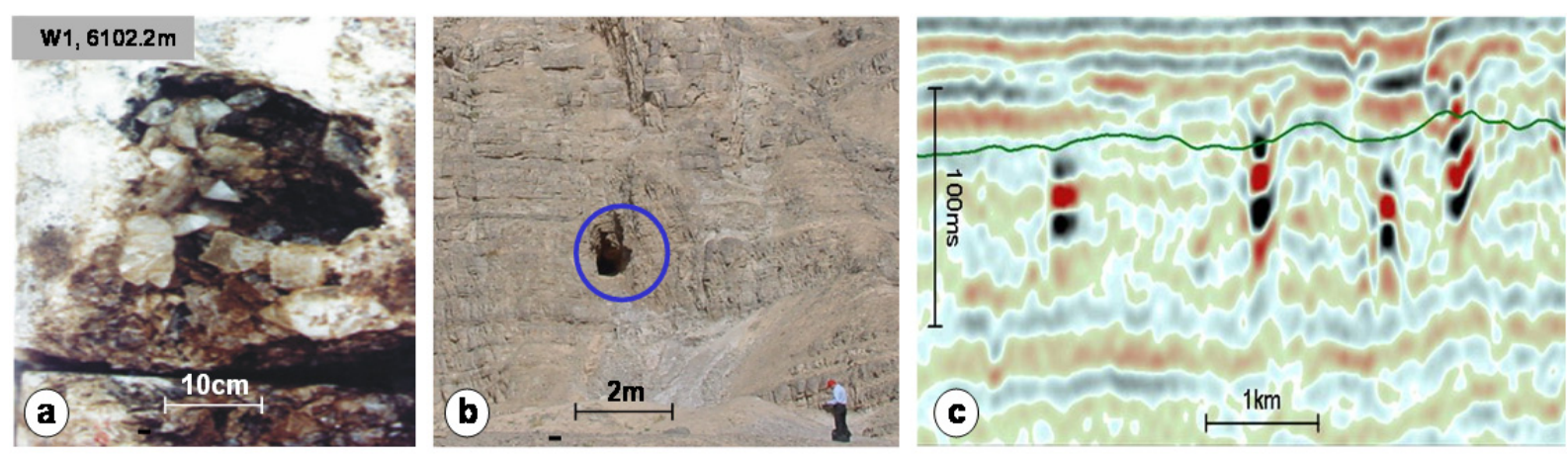

Figure 1. The typical core photo (a), outcrop (b) and post-stack seismic section (c) of the paleokarst carbonate reservoir in the Tarim Basin. The green line in the seismic section (c) is the top of carbonate strata. This secondary reservoir is often characterized by 'bead-like-reflection' in the post-stack section, showing significant strong heterogeneity

For the modeling of this very heterogeneous reservoir, the traditional lateral interpolation and extrapolation of impedance $\operatorname{logs}$ between well locations are obviously unqualified, while the current geological rules such as sedimentary facies of clastic rocks (Damsleth et al., 1992) and weathering karst facies of carbonate rocks (Huo et al., 2007) are insufficient to guide as well. Besides, multiple seismic attributes employed in regressing qualitative 
reservoir predictions (Liu et al., 2010) are contaminated by AVO effects and also not acceptable.

\section{Method}

Different from the conventional modeling methods by directly lateral interpolation and extrapolation of well logs without geological meanings, or adding the guidance with seismic velocities or attributes contaminated by AVO effects, the proposed method in this paper is conducted by referring to the heterogeneous information from the pure P-wave data, which have removed the AVO effects by the pre-stack inversion. As shown in the Figure 2, it contains three major approaches including the pure P-wave data computation, the relative and absolute impedance LFM construction, and the impedance inversion.

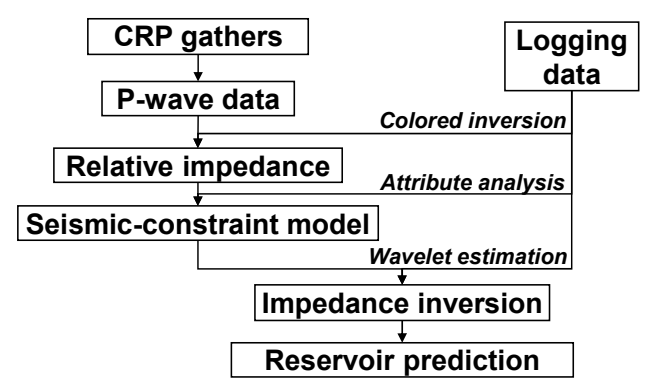

Figure 2. The workflow of the amplitude-based modeling method and its application on the reservoir prediction. The P-wave data computed from CRP gathers by pre-stack inversion have a higher resolution, and are more beneficial for LFM modeling and reservoir prediction

\subsection{P-wave Data Computation}

For the improvement of seismic data SNR in the current multiple-fold acquisition system, it is a common practice to stack all the traces of a common-reflection-point (CRP) as the so-called zero-incident data for interpretation enhancement (Han et al., 2013). However, this process has made a reluctant compromise to the sacrifice of data resolution and accuracy due to the contamination of AVO effects no matter how much SNR degree could be enhanced (Zhang et al., 2013). To overcome this shortcoming, the P-wave data is theoretically inverted in this paper through AVO effects removal via pre-stack AVO inversion. The most useful form of the pre-stack inversion formula (Gidlow et al., 1992) can be reorganized from Aki \& Richards equation and illustrated as follow:

$$
R_{p p}(\theta)=\left(1+\tan ^{2} \theta\right) R_{p}-8 \frac{V_{s}^{2}}{V_{p}^{2}} R_{s} \sin ^{2} \theta-\left(\frac{1}{2} \tan ^{2} \theta-2 \frac{V_{s}^{2}}{V_{p}^{2}} \sin ^{2} \theta\right) \frac{\Delta \rho}{\rho}
$$

Where, Rp and Rs are theoretical zero-offset P-wave reflectivity and S-wave reflectivity respectively,

$$
R_{p}=\frac{1}{2}\left(\frac{\Delta \rho}{\rho}+\frac{\Delta V_{p}}{V_{p}}\right), \quad R_{s}=\frac{1}{2}\left(\frac{\Delta \rho}{\rho}+\frac{\Delta V_{s}}{V_{s}}\right)
$$

And Vp, Vs and $\rho$ are the average P-wave velocity, S-wave velocity and density of the reflection boundary, respectively. $\theta$ is the average of incidence and transmission angles. $\operatorname{Rpp}(\theta)$ is the elastic reflectivity with ray-path of incident angle, while $\Delta \rho / \rho$ is the density gradient. Vs/Vp is the S-to-P-wave velocity ratio.

For the solving of non-linear equation (1), the P-wave result is comparatively stable since the coefficient of Rp is usually bigger than that of Rs or $\Delta \rho / \rho$, and has nothing to do with Vp/Vs (Zhang et al., 2013). The computed Rp data are the pure P-wave data since wavelets are not removed in our inversion process. Comparing to the conventional full-stack data, the computed P-wave data have a higher resolution and are more beneficial for the reservoir prediction (Han et al., 2013; Zhang et al., 2013), and inevitably for the LFM model construction.

\subsection{LFM Construction}

To construct a rational LFM to describe strong heterogeneous reservoirs, the valuable computed pure P-wave data should be fully utilized; yet seismic data affected by the wavelets cannot be directly correlated to the 
impedance. Therefore, the colored inversion (Lancaster \& Whitcombe, 2000) is employed to efficiently achieve the relative impedance (RI). Superior to the conventional band-limited inversion that takes no account of seismic wavelet or calibration to the earth, such as phase rotation, trace integration and recursive inversion, the colored inversion technique implicitly not only accounts for the seismic wavelet but also is consistent with log data. It is easier and faster than more sophisticated techniques, i.e. sparse-spike inversion.

Although the inverted RI has contained the heterogeneity reservoir information, and it has been reported to be effective for the prediction of reservoir properties (Kumar et al., 2014), its relative variations including negative and positive values could hardly be added into the LFM straightway. The best alternative counterpart is the absolute impedance (AI). Theoretically, the AI is represented as the summation of the RI and the low frequency impedance (Figure 3a); while the low frequency impedance often gradually increases with the increase of burial depth, which consequently causes the AI and the RI increasingly uncorrelated (Figure 3c). On the other hand, they are well consistent with each other (Figure 3d) if the low frequency background stays relatively constant (Figure 3b); that is exactly appropriate for the deeply buried paleokarst carbonate strata in the Tarim Basin. Therefore, the absolute impedance LFM away from well locations of this strong heterogeneous reservoir can be transformed from relative impedance, on the basis of their fitted relationships.
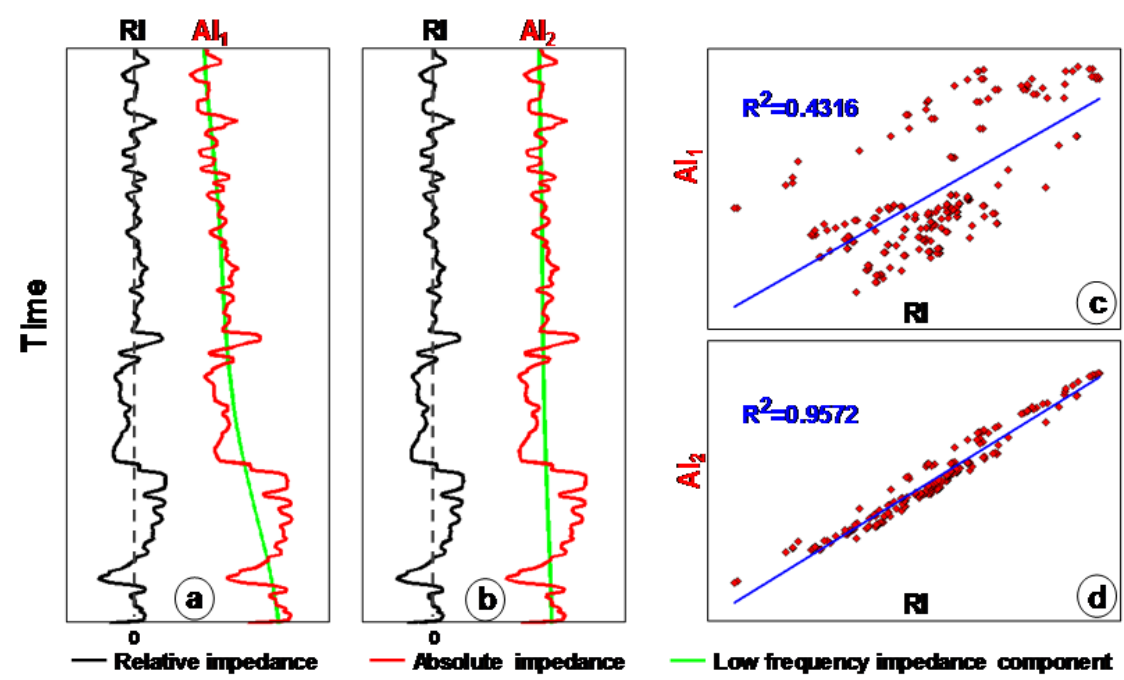

Figure 3. The comparison between the relative impedance (RI) and the absolute impedance (AI) for different low frequency backgrounds. The RI and the AI are badly correlated (c) with each other for the varying (i.e., gradual increasing) impedance background (a), whilst well consistent (d) for the relatively constant (i.e., the carbonate strata in the ZG8 area) background (b)

\subsection{Impedance Inversion}

With the constructed absolute impedance LFM, the seismic inversion that converts seismic traces into acoustic impedances can be conducted; thus the impedance as well as accompanied attributes for instance lithology and porosity can be quantified away from the wells.

\section{Application}

Based on the three qualified partial angle stacks (3-13, 13-23 and 23-33 degree) via pre-stack AVO inversion in ZG8 area, Tazhong Uplifit, the pure P-wave data are achieved to compare with conventional full stack data on reservoir characterization. The pre-stack AVO inversion results demand both high quality input data and high-efficiency inversion algorithms. Successful data processing in this area often focuses on preserving the true amplitudes in the whole processing workflow, e.g., amplitude compensation, noise attenuation, resolution enhancement and pre-stack migration. Significantly, the basic criterion for preserving amplitude is that the AVO characteristics between synthetic and obtained gathers should be similar (Sun et al., 2011; Zhang et al., 2011a; Zhang et al., 2011b; Feng et al., 2012). Meanwhile, the pre-stack seismic inversion should be carefully applied by seriously controlling the quality of four major approaches, including angle partial stack data preparation, wavelet estimation, low-frequency model building and inversion parameter control (Zhang et al., 2011a; Zhang et al., 2011b). Theoretically, the pure P-wave data are prone to be more precisely achieved than other elastic 
parameters, i.e., S-wave data and density data (Zhang et al., 2013).
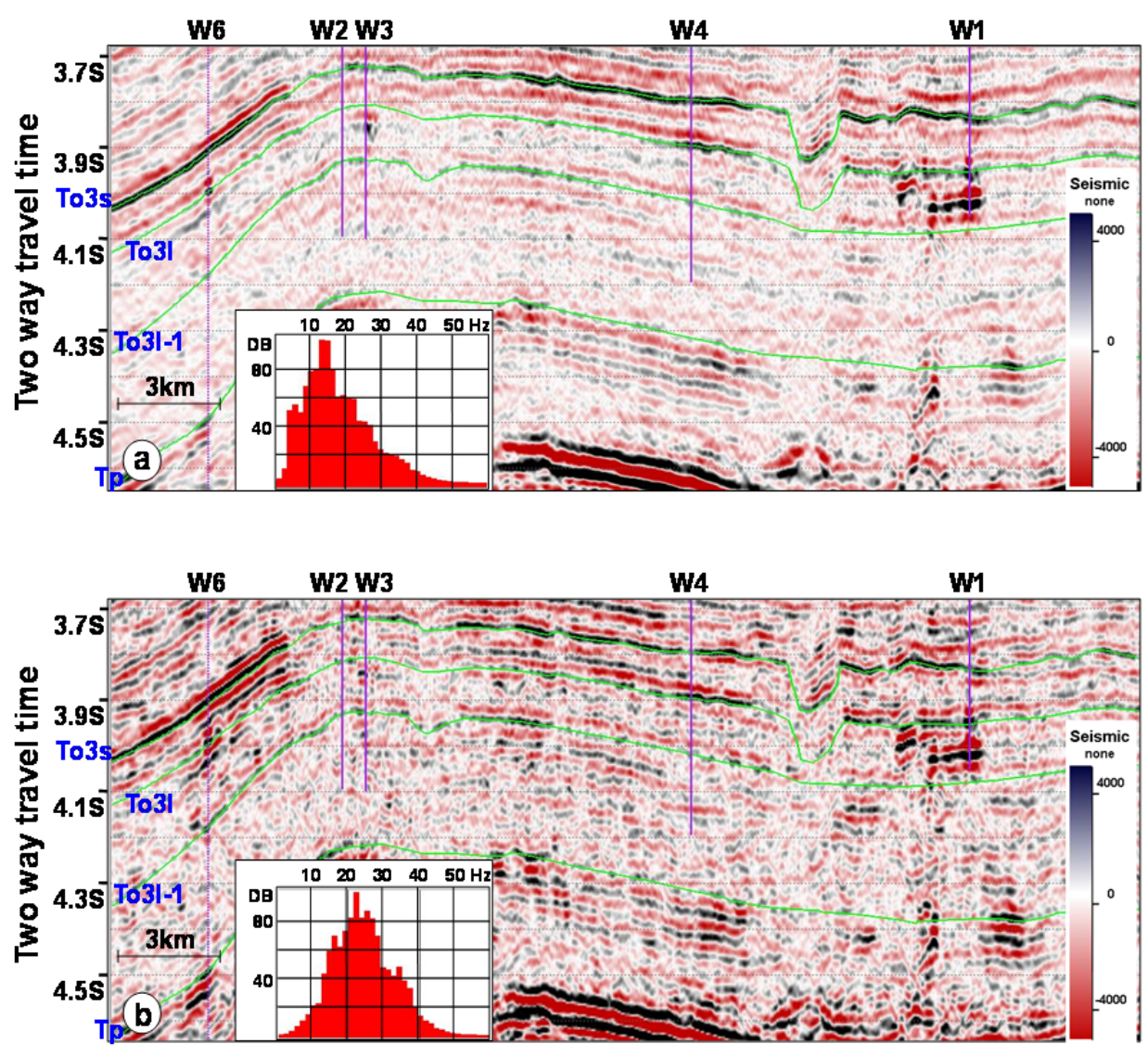

Figure 4. The comparison between the full-stack (a) and the inverted P-wave section (b) across 5 typical wells in the ZG8 area. After the AVO effects removal, the inverted P-wave section has more clear and continuous events, and has $8 \mathrm{~Hz}$ dominant frequency enhanced comparing with the full stack data in the carbonate strata in Ordovician. The To3l and To31-1 are the top and base of the first part of target Yingshan formation, respectively.

The top line indicates the top (To3s) of the carbonate strata

Figure 4 illustrates the comparison between the full-stack (Figure 4a) and the inverted P-wave section (Figure 4b) across 5 typical wells in the ZG8 area, Tarim Basin. The dominant frequency of inverted P-wave data is $24 \mathrm{~Hz}$ in the target Yingshan formation, Ordovician through AVO effects removal, while the opponent from the stack data is only $15 \mathrm{~Hz}$ on the target carbonate strata. Moreover, the reflection events of the pure P-wave data are obviously more continuous comparing with that of the full-stack data. These enhancements are extensively crucial for extracting accurate seismic attributes.

Figure 5 shows the inverted relative impedance across the same section with Figure 4. The well W1, W3, W4 and W6 drilled through good dissolution reservoirs with a good pay, whilst the well W2 is dry. As the operator of colored inversion essentially includes wavelet information (extracted from finite logging and corresponding seismic data), the reservoir's strong heterogeneous characters in the inverted relative impedance (RI) have been not only availably conserved, but also notably highlighted (Figure 5, marked by blue ellipses). This increase is further beneficial for the LFM building and inversion. 


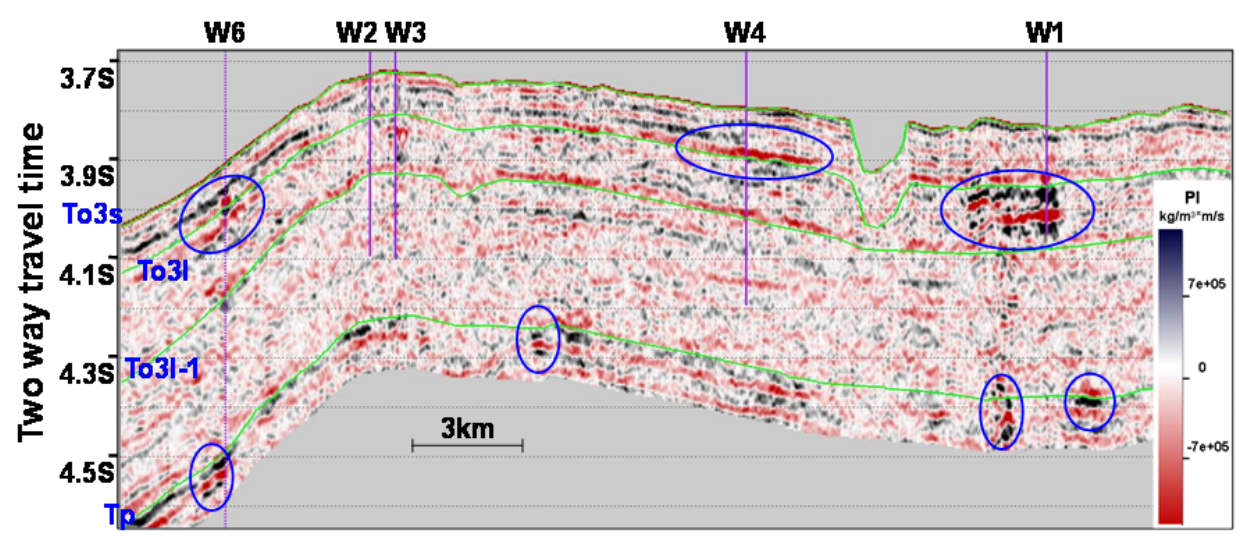

Figure 5. The inverted relative impedance across the same section with Figure 4. The heterogeneous strong amplitudes associated with good reservoirs in the P-wave seismic data are successfully preserved (blue ellipses)

The cross-plot between the measured acoustic impedance (AI) and the computed relative impedance (RI) (Figure 6) at well locations indicates that they are well correlated in two different segments including the reservoir part $\mathrm{AB}$ and the carbonate matrix part $\mathrm{BC}$ ' divided by porosity, respectively. Particularly, the two relationships can be respectively fitted as:

$$
\begin{aligned}
\mathrm{AB}: \mathrm{AI} & =3.556 * \mathrm{RI}+1.828 \mathrm{e}+07 \\
\mathrm{BC}: & \mathrm{AI}=0.121 * \mathrm{RI}+1.656 \mathrm{e}+07
\end{aligned}
$$

Thus, for this heavily compacted carbonate with a relative constant impedance background, the AI can be rationally transferred from RI according to the former analysis. Meanwhile, to mitigate the inversion side-lobes, we use a constant background marked by $\mathrm{BC}$ instead of the relatively steady values of $\mathrm{BC}$ ' in Figure 6 to compute the AI LFM.

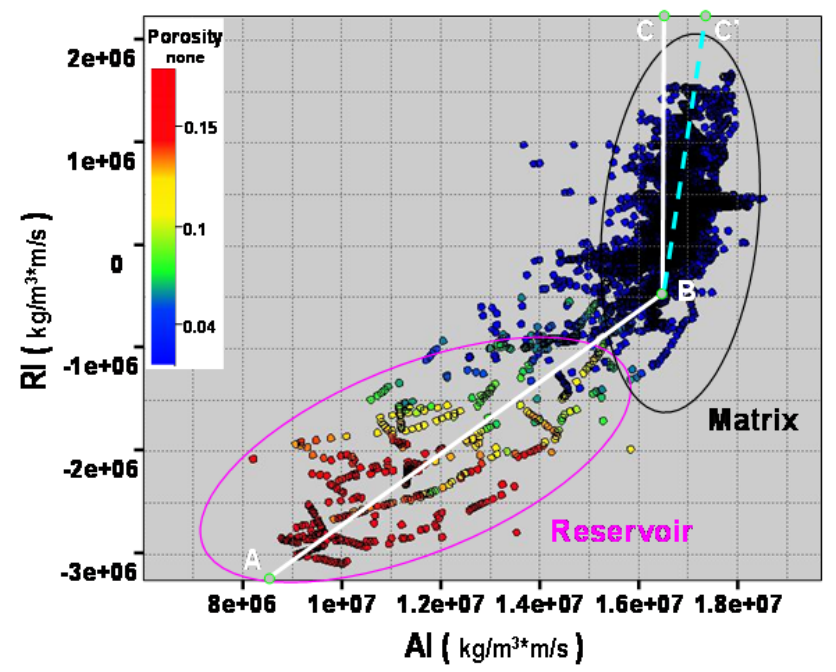

Figure 6. The cross-plot between the measured acoustic impedance (logging) filtered to the seismic-frequency band (AI in $\mathrm{x}$-axis) and the computed relative impedance (RI in y-axis) at well locations 

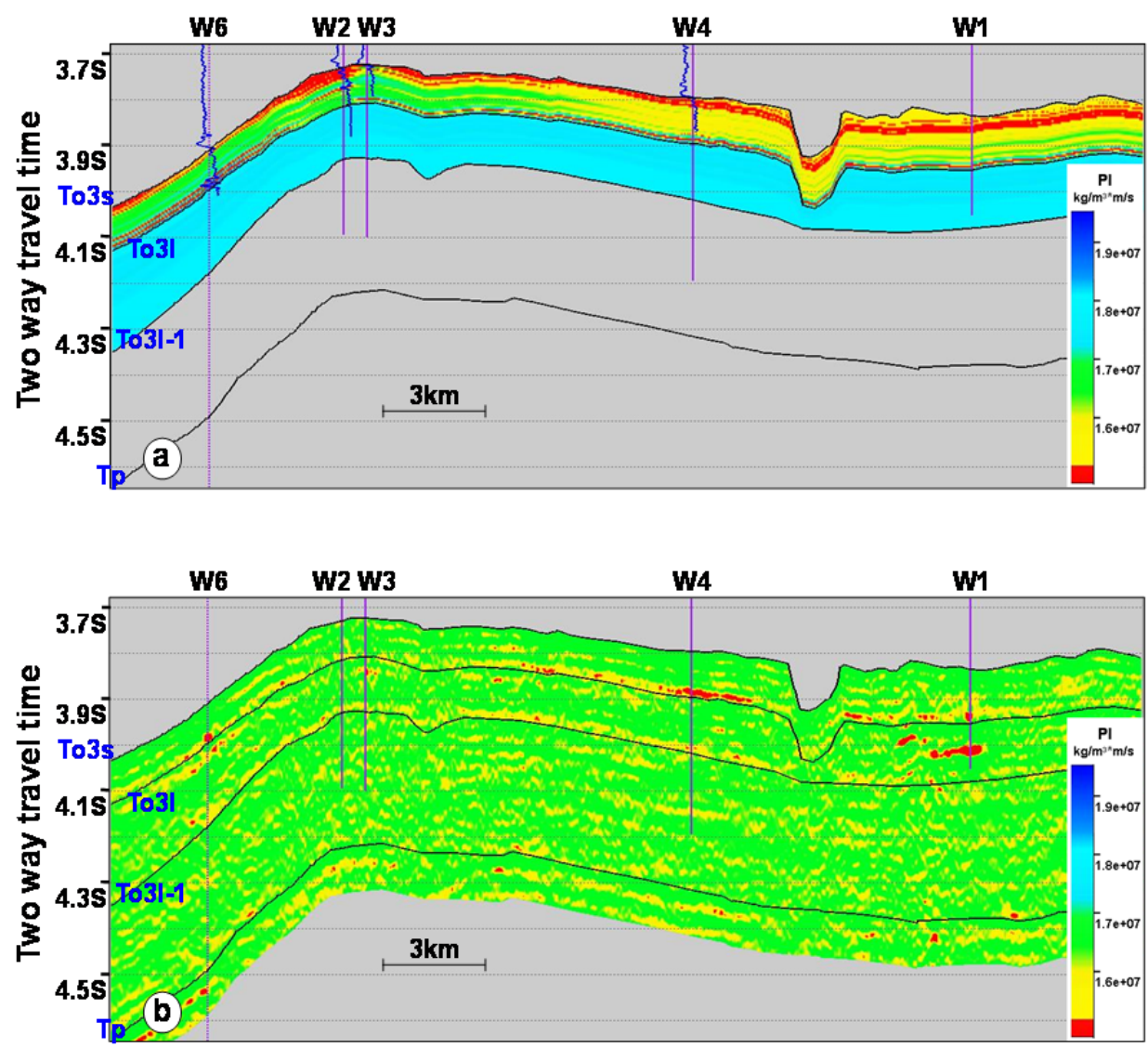

Figure 7. Low frequency models achieved by the traditional interpolation and extrapolation method (a) and the proposed amplitude-based method (b). The blue lines near well locations in Figure 7a indicate the measured acoustic impedances (insufficient for the well W1)

Figure 7 demonstrates the difference between the Low frequency acoustic impedance models constructed by the traditional interpolation and extrapolation method and the proposed amplitude-based method, respectively. For the traditional result in Figure 7a, the heterogeneous carbonate reservoirs in the target Yingshan formation (To31-To31-1) are irrelevantly modeled as homogeneous because of the logging insufficiency, while some fallacious stripped low impedance values caused by the logging inaccuracy near the top carbonate boundary are presented too. On the contrary, a more reasonable AI LFM considerably preserved the heterogeneous reservoir information (Figure 7b) has been successfully constructed by the proposed amplitude-based method.

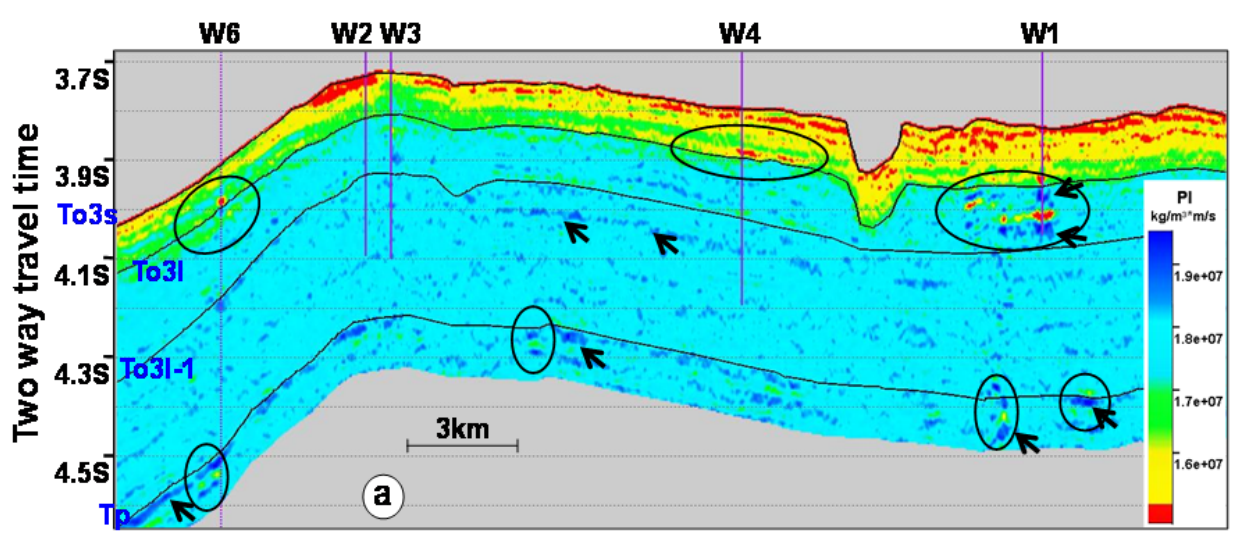




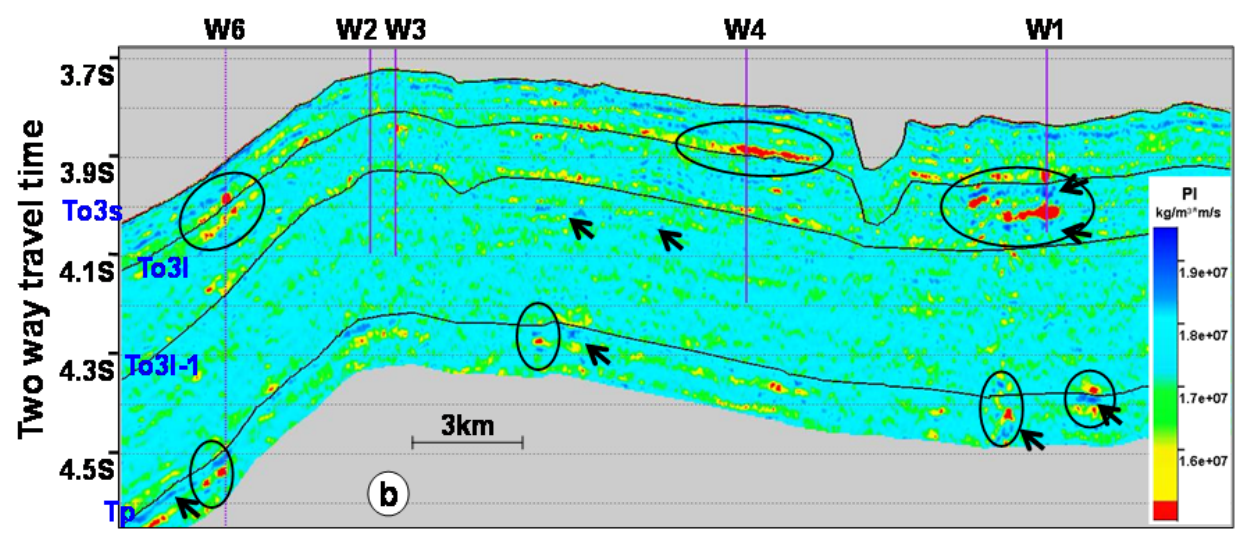

Figure 8. The inverted impedance based on the traditional interpolation and extrapolation modeling method (a) and the proposed amplitude-based modeling method (b)

As the inverted impedance has not only eliminated the wavelet influence but also added the drilling information in the initial earth model, it is widely suggested to be better correlated with the reservoir quality than the seismic amplitude (Zhang et al., 2011a; Yang et al., 2007). We conduct the impedance inversion based on two different LFMs respectively constructed from the traditional and proposed methods. The good carbonate reservoir often renders a lower impedance value comparing with that of the compacted carbonate matrix. As shown in Figure 8a, the stripped contaminations near the top carbonate strata still exist in the traditional result, while the relative impedance variations added into a homogeneous background are relatively reduced and smoothed (marked by black ellipses). Thus, reservoirs tend to be overestimated in the top carbonate strata but underestimated in the rest strata. Contrarily, the new result (Figure 8b) has demonstrated a better description for this heterogeneous reservoir. In particular, the inversion side-lobes near the reservoirs are preferably diminished comparing with the traditional result (the dark blue areas marked by the dark arrowheads).

Spatially, the RMS (root mean square) attributes of the two sets of results are extracted and compared with that of pure P-wave data in Figure 9. Comparing with the section comparisons, the spatial maps of predicted reservoirs are more capable of indicating the reservoir heterogeneity. Obviously, the traditional result (Figure 9b) is controlled by well locations (called as buphthalmos) because of the lateral interpolation and extrapolation, and not consistent with the seismic data. Comparatively, the proposed result (Figure 9c) has successfully preserved the strong heterogeneous reservoir distribution contained in seismic reflections.

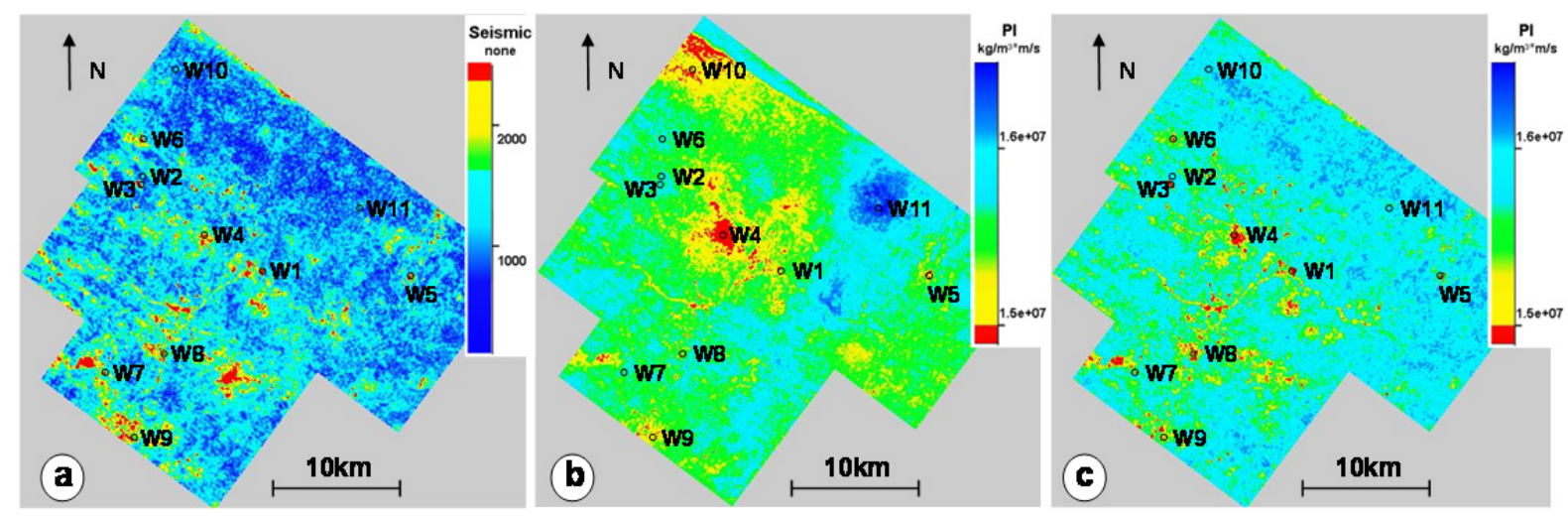

Figure 9. The comparison of the root mean square attribute of the pure P-wave data (a), the inverted impedance based on the traditional modeling (b) and the proposed amplitude-based modeling (c) method, in Yingshan fm, Ordovician, ZG8 area, respectively. Spatial well-dominant effects (called as buphthalmos) caused by the lateral interpolation and extrapolation are obviously exhibited in the traditional result. The new result is comparatively consistent with seismic data 


\section{Conclusion}

The amplitude-based modeling method, that is conducted by utilizing the heterogeneous information from the pure P-wave data to guide the geological model construction, is more suitable for strong heterogeneous reservoirs description especially in well insufficient or absent areas, suggested by the comparisons with the traditional methods in ZG8 area.

\section{Acknowledgements}

The authors thank the NSFC and Sinopec joint key project (U1663207), 973 Program (2014CB239104), and China Postdoctoral Science Foundation for the final support.

\section{References}

Cerney, B., \& Bartel, D. C. (2007). Uncertainties in low-frequency acoustic impedance models. The Leading Edge, 26(1), 74-87. http://dx.doi.org/10.1190/1.2431834

Damsleth, E., Tjolsen, C. B., \& More, H. (1992). A two-stage stochastic model applied to a North Sea reservoir. Journal of Petroleum Technology, 44(4), 402-486. http://dx.doi.org/10.2118/20605-PA.

Feng, X., Wang, Y., Wang, X., Wang, N., Gao, G., \& Zhu, X. (2012). The application of high-resolution 3D seismic acquisition techniques for carbonate reservoir characterization in China. The Leading Edge, 31(2), 168-179. http://dx.doi.org/10.1190/1.3686914

Gidlow, P. M., Smith, G. C., \& Vail, P. J. (1992). Hydrocarbon detection using fluid factor traces: A case study. Proceedings of the Joint SEG/EAEG Summer Research Workshop on "How Useful is Amplitude-Versus-Offset (AVO) Analysis?"(pp. 78-89).

Grant, S. (2013). The impact of low frequency models on reservoir property predictions. Proceedings of the 2013 EAGE Conference \& Exhibition, London, England. http://dx.doi.org/10.3997/2214-4609.20130335

Hampson, D., Russell, B., \& Bankhead, B. (2005). Simultaneous inversion of pre-stack seismic data. Proceedings of the 2005 SEG Conference \& Exhibition, Houston, USA. http://dx.doi.org/10.1190/1.2148008

Han, J., Sun, S., Zhang, X., Zhang, Y., Chen, J., Pan, Y., Liu, X., \& Zhao, H. (2013). Integrated Identification for Complex Reservoir Based on Pure P-Wave Data and Post-Stack Data. Proceedings of the 2013 SEG Conference \& Exhibition, San Antonio, USA. http://dx.doi.org/10.1190/segam2013-1287.1

Huck, A., Quiquerez, G., \& De, G. P. (2010). Improving seismic inversion through detailed low frequency model building. Proceedings of the 2010 EAGE Conference \& Exhibition, Barcelona, Spain. http://dx.doi.org/10.3997/2214-4609.201400776

Huo, C. L., Li, G., Zhao, C. M., Yan, W. P., \& Yang, Q. D. (2007). Integrated reservoir geological modeling based on seismic, log and geological data. Acta Petrolei Sinica, 28(6), 66-71.

Kumar, N., \& Negi, S. (2012). Low-frequency modeling and its impact on seismic inversion data. Proceedings of the 9th Biennial International Conference \& Exposition on Petroleum Geophysics, Hyderabad, India.

Lancaster, S., \& Whitcombe, D. (2000). Fast track 'coloured' inversion. Proceedings of the 2000 SEG Conference \& Exhibition, Calgary, Canada. http://dx.doi.org/10.1190/1.1815711

Liu, L., Sun, Z., Yang, H., Han, J. F., \& Jin, B. (2010). Modeling of facies-controlled carbonate reservoirs in the Tazhong area and its application. Acta Petrolei Sinica, 31(6), 952-958.

Loucks, R. G. (1999). Paleocave carbonate reservoirs: origins, burial-depth modifications, spatial complexity, and reservoir implications. AAPG bulletin, 83(11), 1795-1834.

Pedersen-Tatalovic, R., Uldall, A., \& Jacobsen, N. L. (2008). Event-based low-frequency impedance modeling using well logs and seismic attributes. The Leading Edge, 27(5), 592-603. http://dx.doi.10.1190/1.2919576

Schultz, P. S., Ronen, S., Hattori, M., Mantran, P., \& Corbett, D. (1994). Seismic-guided estimation of log properties (Part 3: A controlled study). The Leading Edge, 13(7), 770-776. http://dx.doi. 10.1190/1.1437036

Sun, S., Wang, H., Liu, Z., Li, Y., Zhou, X., \& Wang, Z. (2012). The theory and application of DEM-Gassmann rock physics model for complex carbonate reservoirs. The Leading Edge, 31(2), 152-158. http://dx.doi.org/10.1190/1.3686912

Sun, S., Zhou, X., Li, Y., Wang, H., \& Zhang, Y. (2011). Fluid Identification of Caved Carbonate Reservoir Based on Prestack Inversion. Proceedings of the 2011 EAGE Conference \& Exhibition, Vienna, Austria. 
http://dx.doi.org/ 10.3997/2214-4609.20149591

Yang, H., Xue, F., Pan, W., Chen, L., Yang, P., Tong, Y., \& Zhao, C. (2010). Seismic Description of Karst Topography And Caves of Ordovician Carbonate Reservoirs Lungu Area Tarim Basin West China.Proceedings of the 2010 SEG Conference \& Exhibition, Denver, USA.http://dx.doi.org/10.1190/1.3513072

Yang, P., liu, Y., Hou, Y., Jia, H., Liu, D., \& Li, H. (2007). Carbonate Reservoirs Prediction Strategy And Technologies In Tarim Basin of China.Proceedings of the 2007 SEG Conference \& Exhibition, San Antonio, USA. http://dx.doi.org/10.1190/1.2793035

Yang, P., Sun, S., Liu, Y., Li, H., Dan, G., \& Jia, H. (2012). Origin and architecture of fractured-cavernous carbonate reservoirs and their influences on seismic amplitudes. The Leading Edge, 31(2), 140-150. http://dx.doi.org/10.1190/1.3686911

Zeng, H., Wang, G., Janson, X., Loucks, R., Xia, Y., Xu, L., \& Yuan, B. (2011). Characterizing seismic bright spots in deeply buried, Ordovician paleokarst strata, Central Tabei uplift, Tarim Basin, Western China. Geophysics, 76(4), 127-137. http://dx.doi.org/10.1190/1.3581199

Zhang, Y., Sun, S., Yang, H., Wang, H., Han, J., Gao, H., \& Jing, B. (2011a). Pre-stack inversion for caved carbonate reservoir prediction: A case study from Tarim Basin, China. Petroleum Science, 8(4), 415-421.

Zhang, Y., Sun, Z., \& Fan, C. (2013). An Iterative AVO Inversion Workflow for S-wave Improvement. Proceedings of the 2013 EAGE Conference \& Exhibition, London, England. http://dx.doi.org/10.3997/2214-4609.20130268

Zhang, Y., Sun, Z., Yang, H., Tang, Z., Wang, H., \& Bai, H. (2011b). The Advantages of Pre-stack Inversion in Heterogeneous Carbonate Reservoir Prediction-A Case Study from Tarim Basin, China. Proceedings of the 2011 EAGE Conference \& Exhibition, Vienna, Austria. http://dx.doi.org/10.3997/2214-4609.20149735

Zou, Z., Ling, Y., Wang, Y., Gao, J., \& Xi, X. Y. (2013). Low-frequency Impedance Modeling Based on Well Logs and Seismic Attributes. Proceedings of the 2013 EAGE Conference \& Exhibition, London, England. http://dx.doi.org/10.3997/2214-4609.20130949

\section{Copyrights}

Copyright for this article is retained by the author(s), with first publication rights granted to the journal.

This is an open-access article distributed under the terms and conditions of the Creative Commons Attribution license (http://creativecommons.org/licenses/by/3.0/). 